\title{
QCD effects in lepton angular distributions of Drell-Yan/Z production and jet discrimination
}

\author{
Wen-Chen Chang, ${ }^{a, *}$ Randall Evan McClellan, ${ }^{b, c}$ Jen-Chieh Peng ${ }^{b}$ and Oleg \\ Teryaev $^{d, e}$ \\ ${ }^{a}$ Institute of Physics, Academia Sinica, Taipei 11529, Taiwan \\ ${ }^{b}$ Department of Physics, University of Illinois at Urbana-Champaign, Urbana, Illinois 61801, USA \\ ${ }^{c}$ Department of Physical Sciences, Pensacola State College, Pensacola, FL 32504, USA \\ ${ }^{d}$ Joint Institute for Nuclear Research, 141980 Dubna, Russia \\ ${ }^{e}$ Dubna International University, 141982 Dubna, Russia \\ E-mail: changwc@phys.sinica.edu.tw, remcclellan@pensacolastate.edu, \\ jcpeng@illinois.edu, teryaev@jinr.ru
}

\begin{abstract}
We present a comparison of data of lepton angular distributions of Drell-Yan/Z production with the fixed-order pQCD calculations by which the baseline of pQCD effects is illustrated. As for the $Z$ production, we predict that $A_{0}$ and $A_{2}$ for $Z$ plus single gluon-jet events are very different from that of $Z$ plus single quark-jet events, allowing a new experimental tool for checking various algorithms which attempt to discriminate quark jets from gluon jets. Using an intuitive geometric approach, we show that the violation of the Lam-Tung relation, appearing at large transversemomentum region, is attributed to the presence of a non-coplanarity effect. This interpretation is consistent with the appearance of violation beyond LO-QCD effect.
\end{abstract}

40th International Conference on High Energy physics - ICHEP2020

July 28 - August 6, 2020

Prague, Czech Republic (virtual meeting)

\footnotetext{
${ }^{*}$ Speaker
} 


\section{Introduction}

Measuring lepton angular distributions of Drell-Yan (D-Y) process [1] provides a powerful tool to explore the reaction mechanisms and the parton distributions of colliding hadrons. For example, the Lam-Tung (L-T) relation [2] has been proposed as a benchmark of the perturbative QCD (pQCD) effect in D-Y process. Violations of L-T relation were observed in the measurements of D-Y production by the fixed-target experiments as well as $\gamma^{*} / Z$ production by the CMS [3] and ATLAS [4] experiments at LHC. It is important to understand the origin of these violations.

It is found that the violation of the L-T relation seen in CMS and ATLAS data in the region of transverse momentum $\left(q_{T}\right)$ greater than $5 \mathrm{GeV}$ could be well described taking into account NNLO pQCD effect [5]. On the other hand, the agreement is not as good in a similar comparison [6,7] for the fixed-target data of NA10 [8], E615 [9] and E866 [10] at $q_{T}<3 \mathrm{GeV}$. Transverse-momentum dependent Boer-Mulders functions [11], correlating the nucleon spin with the intrinsic transverse momentum of partons, have been suggested to account for a violation of the L-T relation observed at small $q_{T}$ in the fixed-target experiments.

In this proceedings, we show that the $q_{T}$ dependence of the angular distribution coefficients, as well as the violation of the Lam-Tung violation, could be obtained if the angular distribution coefficients were analyzed as a function of the number of accompanying jets in $Z$-boson production measured by the CMS and ATLAS Collaborations [12]. Furthermore we compare the data of dilepton angular parameters $\lambda, \mu, v$ and the L-T violation quantity $1-\lambda-2 v$ measured by E615 [9] with the fixed-order pQCD calculations. Finally we interpret some notable features of pQCD results using the geometric model [13-15]. More results and greater details can be found in Ref. [7, 12].

\section{Lepton angular distributions of $Z$ production and jet discrimination}

The lepton angular distribution in the $Z$ rest frame can be expressed as [3, 4]

$$
\begin{aligned}
\frac{d \sigma}{d \Omega} & \propto\left(1+\cos ^{2} \theta\right)+\frac{A_{0}}{2}\left(1-3 \cos ^{2} \theta\right)+A_{1} \sin 2 \theta \cos \phi+\frac{A_{2}}{2} \sin ^{2} \theta \cos 2 \phi \\
& +A_{3} \sin \theta \cos \phi+A_{4} \cos \theta+A_{5} \sin ^{2} \theta \sin 2 \phi+A_{6} \sin 2 \theta \sin \phi+A_{7} \sin \theta \sin \phi,
\end{aligned}
$$

where $\theta$ and $\phi$ are the polar and azimuthal angles of leptons in the rest frame of $Z$. Since the intrinsic transverse momenta of the annihilating quark and antiquark is neglected in the original Drell-Yan model, the angular distribution is simply $1+\cos ^{2} \theta$ and all angular distribution coefficients, $A_{i}$, vanish. For a non-zero dilepton transverse momentum, $q_{T}$, these coefficients can deviate from zero due to QCD effects. However, it was predicted that the coefficients $A_{0}$ and $A_{2}$ should remain identical, $A_{0}=A_{2}$, i.e. the Lam-Tung relation [2].

Figure 1 shows the CMS data for $A_{0}$ and $A_{0}-A_{2}$. Pronounced $q_{T}$ dependence of $A_{0}$ is observed and the Lam-Tung relation, $A_{0}-A_{2}=0$, is clearly violated. There are two NLO QCD subprocesses for $Z$ production: $q \bar{q} \rightarrow Z g$ annihilation process, and $q g \rightarrow Z q$ quark Compton scattering process. In the Collins-Soper frame [16], the NLO pQCD predictions of $A_{0}$ and $A_{2}$ as a function of $q_{T}$ of $Z$ for these two processes are $A_{0}=A_{2}=q_{T}^{2} /\left(M_{Z}^{2}+q_{T}^{2}\right)$ [17] and $A_{0}=A_{2}=5 q_{T}^{2} /\left(M_{Z}^{2}+5 q_{T}^{2}\right)[18,19]$, respectively. The dotted and dashed curves in Fig. 1(a) correspond to these NLO expressions.

As the $q \bar{q}$ and $q g$ processes contribute to the $p p \rightarrow Z X$ reaction incoherently, the observed $q_{T}$ dependence of $A_{0}$ reflects the combined effect of these two contributions. A best-fit to the CMS 

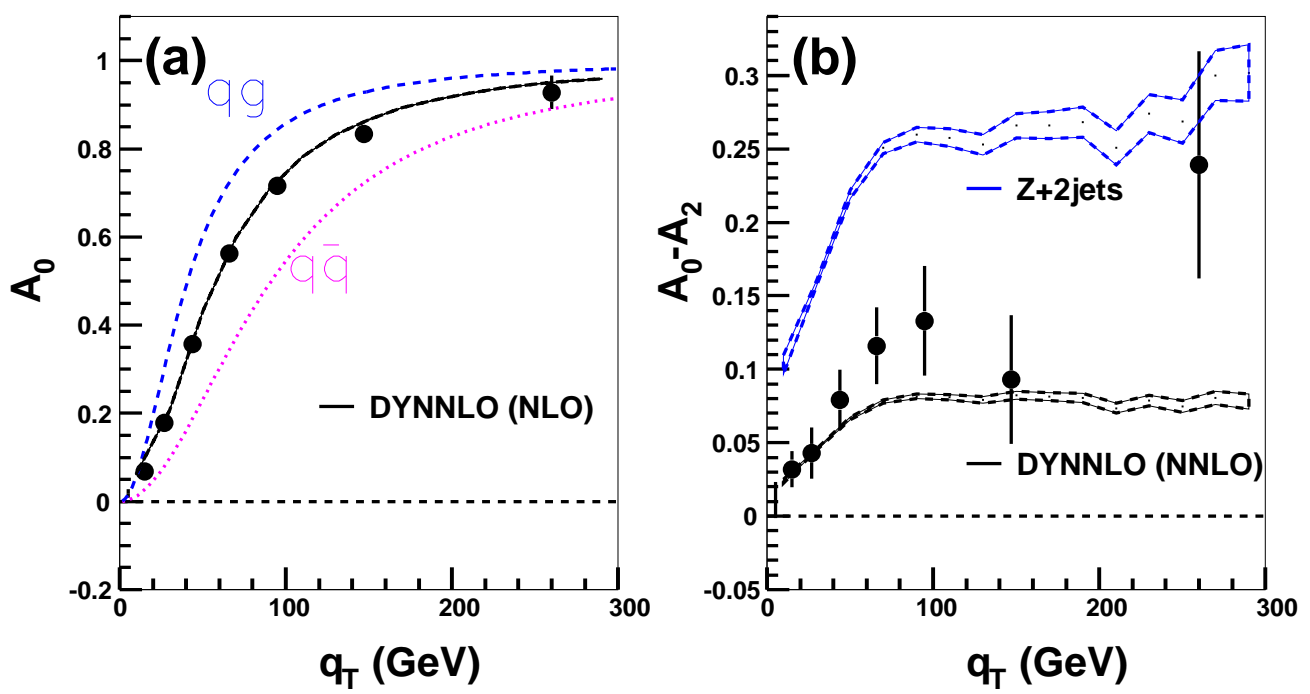

Figure 1: Comparison between the CMS data [3] of $A_{0}$ and $A_{0}-A_{2}$ for $Z$ production from $p-p$ collisions with fixed-order pQCD calculations. Curves correspond to calculations described in the text.

$A_{0}$ data, shown as the solid line in Fig. 1(a), gives a mixture of $58.5 \pm 1.6 \% q g$ and $41.5 \pm 1.6 \% q \bar{q}$ processes. For $p p$ collisions at the LHC, the $q g$ process is expected to be more important than the $q \bar{q}$ process, in agreement with the best-fit result. For $Z$ plus single-jet events, Fig. 1(a) shows that there is remarkable difference in the $q_{T}$ dependence for $A_{0}$ between the $q \bar{q}$ annihilation process and the $q g$ Compton process. Since it is a high- $p_{T}$ gluon (quark) jet associated with the $q \bar{q}(q g)$ process at the $\alpha_{s}$ level, one could first utilize the existing algorithms for quark (gluon) jet identification to separate the $q \bar{q}$ annihilation events from the $q g$ Compton events and investigate the angular distribution of individual event samples. Their angular distribution coefficients for $Z$ plus single jet data would also provide a powerful tool for testing various algorithms designed to distinguish quark jets from gluon jets.

For the $Z$ plus multi-jet data, the L-T relation is expected to be violated at a higher level than that of the inclusive production data. Exclusion of the $Z$ plus single-jet events satisfying the L-T relation, would enhance the violation of the L-T relation. We have carried out pQCD calculations using DYNNLO [20,21] to demonstrate this effect. Figure 1(b) compares the DYNNLO calculations with the CMS $A_{0}-A_{2}$ data. The black band corresponds to the NNLO calculation including contributions from the events of $Z$ with single jet and two jets. The blue band singles out the contributions to $A_{0}-A_{2}$ from $Z$ plus two jets only, showing that the violation of the Lam-Tung relation is indeed amplified for the multi-jet events. This can be readily tested with the data collected at the LHC.

\section{Lepton angular distributions of Drell-Yan production in fixed-target experiments}

In the rest frame of the virtual photon in the D-Y process, another expression for the lepton angular distributions commonly used by the fixed-target experiments is given as [2]

$$
\frac{d \sigma}{d \Omega} \propto 1+\lambda \cos ^{2} \theta+\mu \sin 2 \theta \cos \phi+\frac{v}{2} \sin ^{2} \theta \cos 2 \phi,
$$


where $\theta$ and $\phi$ refer to the polar and azimuthal angles of leptons. The $\lambda, \mu, v$ are related to $A_{0}, A_{1}, A_{2}$ in Eq. (1) via

$$
\lambda=\frac{2-3 A_{0}}{2+A_{0}} ; \quad \mu=\frac{2 A_{1}}{2+A_{0}} ; \quad v=\frac{2 A_{2}}{2+A_{0}} .
$$

Equation (3) shows that the L-T relation, $1-\lambda-2 v=0$, is equivalent to $A_{0}=A_{2}$.

\section{$\mathrm{E} 615 \pi^{-}+\mathrm{W}$ at $252 \mathrm{GeV}$}
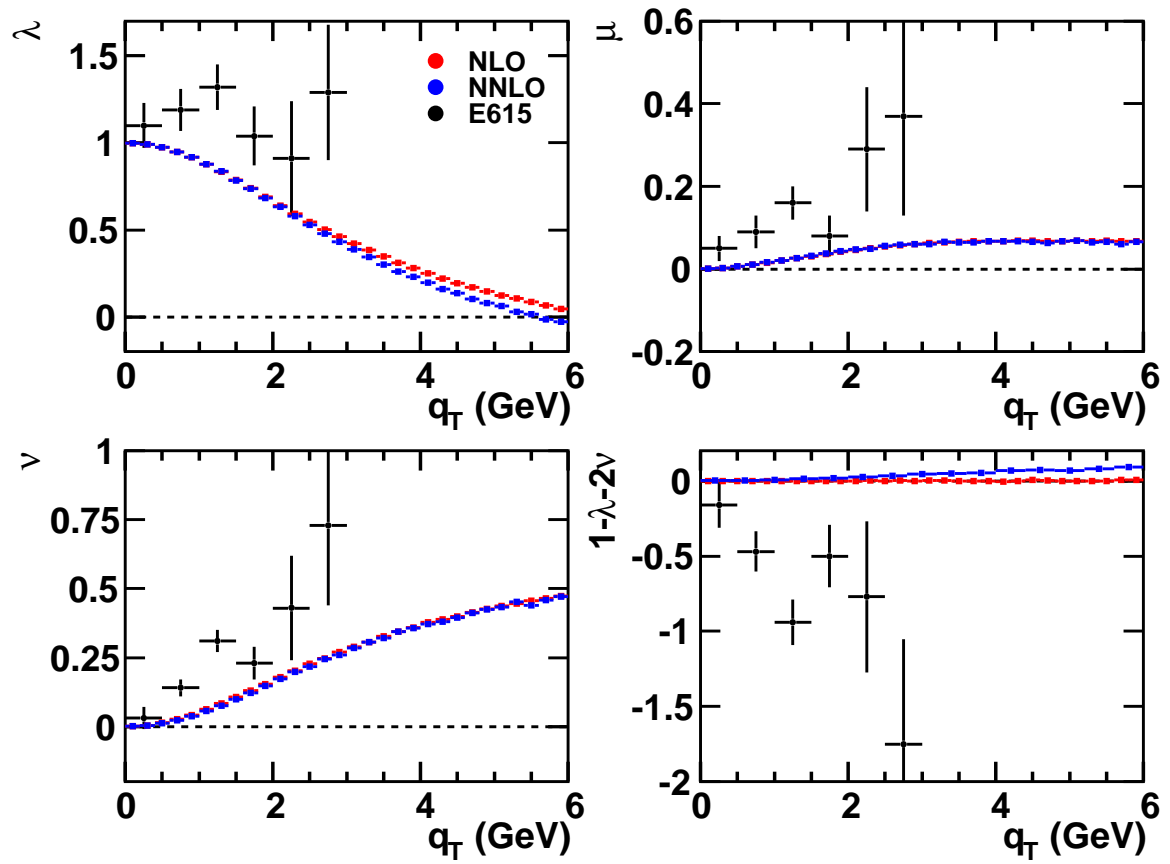

Figure 2: Comparison of NLO (red points) and NNLO (blue points) fixed-order pQCD calculations with the $\mathrm{E} 615 \pi^{-}+W \mathrm{D}-\mathrm{Y}$ data at $252 \mathrm{GeV}$ [9] (black points) for $\lambda, \mu, v$ and $1-\lambda-2 v$.

In Fig. 2, we compare the results of $\lambda, \mu, v$, and the L-T violation, $1-\lambda-2 v$, from the fixed-order pQCD calculations with 252-GeV $\pi^{-}+W$ data from E615 experiment [9]. The angular parameters are evaluated as a function of $q_{T}$ in the Collins-Soper frame. Overall, the calculated $\lambda$, $\mu$ and $v$ exhibit distinct $q_{T}$ dependencies.

At $q_{T} \rightarrow 0, \lambda, \mu$ and $v$ approach the values predicted by the collinear parton model: $\lambda=1$ and $\mu=v=0$. As $q_{T}$ increases, Fig. 2 shows that $\lambda$ decreases toward its large- $q_{T}$ limit of $-1 / 3$ while $v$ increases toward $2 / 3$, for both $q \bar{q}$ and $q G$ processes $[18,19]$. The $q_{T}$ dependence of $\mu$ is relatively mild compared to $\lambda$ and $v$. This is understood as a result of some cancellation effect, to be discussed in Sec. 4.

Comparing the results of the NLO with the NNLO calculations, $\lambda(\mathrm{NNLO})$ is smaller than $\lambda(\mathrm{NLO})$ while $\mu$ and $v$ are very similar for NLO and NNLO. The amount of L-T violation, $1-\lambda-2 v$, is zero in the NLO calculation, and nonzero and positive in the NNLO calculation. As seen in Fig. 2, the pQCD predicts a sizable magnitude for $v$, comparable to the data. Such pQCD effect should be included in the determination of nonperturbative Boer-Mulders effect from the data of $v$. 


\section{Geometric model}

As introduced above, both CMS and E615 data of lepton angular distributions for $Z$ and D-Y production can be reasonably well described by the NLO and NNLO pQCD calculations. It is interesting to see that various salient features of $\mathrm{pQCD}$ calculations could be understood using a geometric approach developed in Refs. [13, 14].

In the Collins-Soper $\gamma^{*} / Z$ rest frame, the hadron plane, the quark plane, and the lepton plane of collision geometry are defined $[13,14]$. A pair of collinear $q$ and $\bar{q}$ with equal momenta annihilate into a $\gamma^{*} / Z$. The momentum unit vector of $q$ is defined as $\hat{z}^{\prime}$, and the quark plane is formed by the $\hat{z}^{\prime}$ and the $\hat{z}$ axes of Collins-Soper frame. The angular coefficients $A_{i}$ in Eq. (3) can be expressed in term of $\theta_{1}$ and $\phi_{1}$ as follows:

$$
A_{0}=\left\langle\sin ^{2} \theta_{1}\right\rangle, \quad A_{1}=\frac{1}{2}\left\langle\sin 2 \theta_{1} \cos \phi_{1}\right\rangle, \quad A_{2}=\left\langle\sin ^{2} \theta_{1} \cos 2 \phi_{1}\right\rangle,
$$

where the $\theta_{1}$ and $\phi_{1}$ are the polar and azimuthal angles of the natural quark axis $\hat{z}^{\prime}$ of the quark plane in the Collins-Soper frame.

Up to NLO $\left(O\left(\alpha_{S}\right)\right)$ in pQCD, the quark plane coincides with the hadron plane and $\phi_{1}=0$. Therefore $A_{0}=A_{2}$ or $1-\lambda-2 v=0$, i.e., the L-T relation is satisfied. Higher order $\mathrm{PQCD}$ processes allow the quark plane to deviate from the hadron plane, i.e., $\phi_{1} \neq 0$. This acoplanarity effect leads to a violation of the L-T relation. For a nonzero $\phi_{1}$, Eq. (4) shows that $A_{2}<A_{0}$. Therefore, when the L-T relation is violated, $A_{0}$ must be greater than $A_{2}$ or, equivalently, $1-\lambda-2 v>0$. This expectation of $1-\lambda-2 v>0$ in this geometric approach agrees with the results of NNLO pQCD calculations shown in Fig. 2. The geometric approach offers a simple and intuitive interpretation for this result.

Furthermore the sign of $\mu$ could be either positive or negative, depending on which parton and hadron the gluon is emitted from [7, 14]. Hence, one expects some cancellation effects for $\mu$ among contributions from various processes. Each process is weighted by the corresponding parton density distributions. At mid-rapidity, the momentum fraction carried by the beam parton is comparable to that of the target parton. Therefore, the weighting factors for various processes are of similar magnitude and the cancellation effect could be very significant, resulting in a small value of $\mu$.

\section{Summary}

We have presented a comparison of the measurements of the angular parameters $A_{0}$ and $A_{0}-A_{2}$ of the $Z$ production from the CMS experiment as well as $\lambda, \mu, v$ and $1-\lambda-2 v$ of the D-Y process from the fixed-target E615 experiment with the corresponding results from the NLO and NNLO pQCD calculations. Qualitatively the transverse momentum dependence of measured angular parameters could be described by pQCD. The L-T violation part $A_{0}-A_{2}$ or $1-\lambda-2 v$ remains zero in the NLO pQCD calculation and turns positive in NNLO pQCD. The measurement of $A_{0}$ and $A_{2}$ coefficients in $Z$ plus single-jet or multi-jet events would provide valuable insights on the origin of the violation of the L-T relation and could be used an an index in discriminating the intrinsic property of high- $q_{T}$ jets. 
Within the geometric picture, the occurrence of acoplanarity between the quark plane and the hadron plane $\left(\phi_{1} \neq 0\right)$, for the PQCD processes beyond NLO offers an interpretation of a violation of the L-T relation. The predicted positive value of $1-\lambda-2 v$, or $A_{0}>A_{2}$ when $\phi_{1}$ is nonzero, is consistent with the NNLO pQCD results.

\section{References}

[1] J. C. Peng and J. W. Qiu, Prog. Part. Nucl. Phys. 76, 43 (2014).

[2] C. S. Lam and W. K. Tung, Phys. Rev. D 21, 2712 (1980).

[3] CMS Collaboration, V. Khachatryan et al., Phys. Lett. B 750, 154 (2015).

[4] ATLAS Collaboration, G. Aad et al., JHEP 08, 159 (2016).

[5] R. Gauld, A. Gehrmann-De Ridder, T. Gehrmann, E. W. N. Glover and A. Huss, JHEP 1711, 003 (2017).

[6] M. Lambertsen and W. Vogelsang, Phys. Rev. D 93, 114013 (2016).

[7] W. C. Chang, R. E. McClellan, J. C. Peng and O. Teryaev, Phys. Rev. D 99, 014032 (2019).

[8] NA10 Collaboration, S. Falciano et al., Z. Phys. C 31, 513 (1986); M. Guanziroli et al., Z. Phys. C 37, 545 (1988).

[9] E615 Collaboration, J. S. Conway et al., Phys. Rev. D 39, 92 (1989); J. G. Heinrich et al., Phys. Rev. D 44, 1909 (1991).

[10] E866/NuSea Collaboration, R. S. Towell et al., Phys. Rev. D 64, 052002 (2001).

[11] D. Boer, Phys. Rev. D 60, 014012 (1999).

[12] J. C. Peng, W. C. Chang, R. E. McClellan and O. Teryaev, Phys. Lett. B 797, 134895 (2019).

[13] J. C. Peng, W. C. Chang, R. E. McClellan, and O. Teryaev, Phys. Lett. B 758, 384 (2016).

[14] W. C. Chang, R. E. McClellan, J. C. Peng and O. Teryaev, Phys. Rev. D 96, 054020 (2017).

[15] J. C. Peng, D. Boer, W. C. Chang, R. E. McClellan and O. Teryaev, Phys. Lett. B 789, 356 (2019).

[16] J. C. Collins and D. E. Soper, Phys. Rev. D16, 2219 (1977).

[17] J. C. Collins, Phys. Rev. Lett. 42, 291 (1979).

[18] R. L. Thews, Phys. Rev. Lett. 43, 987 (1979).

[19] J. Lindfors, Phys. Scr. 20, 19 (1979).

[20] S. Catani and M. Grazzini, Phys. Rev. Lett. 98, 222002 (2007).

[21] S. Catani et al., Phys. Rev. Lett. 103, 082001 (2009). 